\title{
Topical application of magnesium to prevent intubation-related sore throat in adult surgical patients: a systematic review and meta-analysis
}

\section{Application topique de magnésium pour prévenir les maux de gorge liés à l'intubation chez les patients chirurgicaux adultes: revue systématique et méta-analyse}

\author{
Akira Kuriyama, MD, MPH (1) · Hirokazu Maeda, MD • Rao Sun, MD, PhD \\ Received: 20 December 2018/Revised: 4 February 2019/Accepted: 3 March 2019/Published online: 22 May 2019 \\ (C) Canadian Anesthesiologists' Society 2019
}

\begin{abstract}
Background Postoperative sore throat negatively affects patient satisfaction and recovery. We conducted a systematic review and meta-analysis to examine the efficacy of preoperative topical administration of magnesium sulfate in preventing postoperative sore throat in adult patients.

Methods We searched Medline, EMBASE, China National Knowledge Infrastructure, and the Cochrane Central Register of Controlled Trials from inception to 6 October, 2018. We included randomized-controlled trials that assessed the efficacy and safety of topical application of magnesium preoperatively in adult patients who underwent endotracheal intubation for general anesthesia. We then pooled the data using a random-
\end{abstract}

This article is accompanied by an editorial. Please see Can J Anesth 2019; 66: this issue.

Electronic supplementary material The online version of this article (https://doi.org/10.1007/s12630-019-01396-7) contains supplementary material, which is available to authorized users.

A. Kuriyama, MD, MPH ( $\square)$

Emergency and Critical Care Center, Kurashiki Central Hospital,

Miwa, Kurashiki, Okayama 710-8602, Japan

e-mail: akira.kuriyama.jpn@gmail.com

H. Maeda, MD

Department of Emergency Medicine, Sugita Genpaku Memorial Obama Municipal Hospital, Fukui, Japan

R. Sun, MD, $\mathrm{PhD}$

Department of Anesthesiology, Tongji Hospital, Tongji Medical College, Huazhong University of Science and Technology,

Hubei, China effects model and conducted a trial sequential analysis on the incidence of sore throat. Our primary outcome was the incidence of sore throat at $24 \mathrm{hr}$ after surgery/extubation. Our secondary outcomes included the severity of sore throat at $24 \mathrm{hr}$ after surgery/extubation and adverse events. Results Eleven randomized-controlled trials involving 1,096 patients were included in this study. Topical application of magnesium was associated with reduced incidence of postoperative sore throat (risk ratio, 0.31; $95 \%$ confidence interval [CI], 0.21 to 0.45) as well as reduced severity of postoperative sore throat (standardized mean difference, - 2.66; 95\% CI, - 3.89 to - 1.43). Three studies reported that significant adverse events were not associated with topical magnesium. The trial sequential analysis suggested that there is adequate evidence supporting the efficacy of topical magnesium in preventing postoperative sore throat.

Conclusion Our study suggests that preoperative topical magnesium can effectively prevent postoperative sore throat.

Trial registration PROSPERO (CRD42018110019); registered 26 September, 2018.

\section{Résumé}

Contexte Les maux de gorge postopératoires ont un impact négatif sur la satisfaction et la récupération des patients. Nous avons réalisé une revue systématique et une méta-analyse afin d'examiner l'efficacité d'une administration topique préopératoire de sulfate de magnésium pour prévenir les maux de gorge postopératoires chez les patients adultes.

Méthode Nous avons effectué une recherche dans les bases de données Medline, EMBASE, China National 
Knowledge Infrastructure et Cochrane Central Register of Controlled Trials de leur création au 6 octobre 2018. Nous avons inclus les études randomisées contrôlées ayant évalué l'efficacité et l'innocuité de l'application topique préopératoire de magnésium chez des patients adultes subissant une intubation endotrachéale pour l'anesthésie générale. Nous avons ensuite mis les données en commun à l'aide d'un modèle à effets aléatoires et réalisé une analyse séquentielle d'essai sur l'incidence des maux de gorge. Notre critère d'évaluation principal était l'incidence de maux de gorge à 24 h après la chirurgie / l'extubation. Nos critères d'évaluation secondaires comprenaient la sévérité des maux de gorge à 24 h après la chirurgie / l'extubation et les événements indésirables.

Résultats Onze études randomisées contrôlées portant sur 1096 patients ont été incluses dans cette étude. L'application topique de magnésium a été associée à une incidence réduite de maux de gorge postopératoires (risque relatif, 0,31; intervalle de confiance [IC] $95 \%, 0,21$ à $0,45)$ ainsi qu'à une réduction de la sévérité des maux de gorge postopératoires (différence moyenne normalisée, - 2,66; IC $95 \%,-3,89$ à - 1,43). Trois études ont rapporté que les événements indésirables importants observés n'étaient pas associés au magnésium en application topique. Selon l'analyse séquentielle de l'essai, les données probantes soutenant l'efficacité du magnésium en application topique pour prévenir les maux de gorge postopératoires sont adéquates.

Conclusion Selon les résultats de notre étude, l'application préopératoire topique de magnésium est efficace pour prévenir les maux de gorge postopératoires.
Enregistrement
de
l'étude PROSPERO

(CRD42018110019); enregistrée le 26 septembre 2018.

Patient satisfaction is a key element of perioperative care. Post-intubation sore throat has a prevalence of up to $68 \%,{ }^{1-6}$ making it a leading undesirable patient outcome affecting patient satisfaction and recovery., 5,8 Postoperative sore throat can affect patient quality of life after hospital discharge ${ }^{7}$ and thus should be prevented whenever possible.

Proposed etiologies of postoperative airway morbidity include mucosal trauma, erosion, and inflammation due to endotracheal intubation. ${ }^{9-13}$ Accordingly, studies have suggested that anti-inflammatory agents such as inhaled, ${ }^{14}$ topical, ${ }^{15}$ or intravenous ${ }^{16}$ corticosteroids, topical benzydamine hydrochloride, ${ }^{17}$ and topical liquorice, ${ }^{18}$ may be able to prevent postoperative sore throat.
Magnesium has been used to alleviate postoperative pain via various routes of administration. Some evidence, though still controversial, suggests that adjuvant perioperative magnesium reduces postoperative pain and analgesic requirements. ${ }^{19-23}$ Magnesium is presumed to exert its antinociceptive effects by inhibiting calcium entry into cells, thereby blocking N-methyl-D-aspartate-type (NMDA) glutamate receptors. ${ }^{24,25}$ Activation of peripheral NMDA receptors may contribute to masticatory muscle, cutaneous, and deep tissue pain. ${ }^{26-29}$ The magnesium in a magnesium sulfate solution is easily ionized, which allows it to be locally absorbed and used by surrounding tissues. Given that NMDA receptors exist both centrally and peripherally, ${ }^{30}$ topical administration of magnesium may counter the nociceptive stimuli caused by mucosal inflammation due to tracheal intubation. Several randomized-controlled trials have examined the efficacy and safety of topical magnesium in preventing postoperative sore throat. Nevertheless, to our knowledge, no systematic review has been performed to assess the weight of the evidence for topical magnesium use.

Hence, we conducted a systematic review and metaanalysis of studies that examined the efficacy and safety of preoperative topical application of magnesium in preventing postoperative sore throat in adults undergoing tracheal intubation for general anesthesia.

\section{Methods}

We followed the standards described in the PRISMA statement ${ }^{31}$ for the reporting of this systematic review. Our protocol was registered at PROSPERO (CRD42018110019).

Eligibility criteria

Study eligibility

We included randomized-controlled studies that compared preoperative topical application of magnesium in adults who underwent surgery under general anesthesia. We excluded quasi-randomized trials and observational or nonrandomized studies.

\section{Patient eligibility}

We included surgical patients aged $\geq 18 \mathrm{yr}$ who underwent endotracheal intubation for surgery under general anesthesia. We excluded studies that used a laryngeal mask airway for patients. We also excluded studies that examined patients undergoing head and neck surgery because such patients have increased levels of postoperative sore throat ${ }^{32-34}$ that would be clinically 
difficult to differentiate from pain related to the surgical site.

\section{Intervention eligibility}

We included studies that intervened by topically applying magnesium to the laryngopharynx to prevent postoperative sore throat. We a priori knew that magnesium could be administrated as a lozenge or via nebulization or gargle, and we placed no restriction on the form of administrated magnesium. We placed no restrictions on the dose or number of doses of magnesium as long as it was instituted preoperatively.

The comparators included non-analgesic methods or active controls that were initiated preoperatively. Nonanalgesic methods included usual care, no pre-treatment, placebo, or the use of agents without any known analgesic potency, such as water. ${ }^{35}$ Active controls included drugs with known prophylactic effects against postoperative sore throat, such as topical ketamine. We placed no restriction on the dose or number of doses of the comparators. We excluded studies that postoperatively administered nonanalgesic methods or active controls.

\section{Outcome measure eligibility}

Our primary outcome was the incidence of postoperative sore throat at $24 \mathrm{hr}$ after surgery/extubation. Because there is no established definition for postoperative sore throat, we accepted the definition used in each study. Studies related to postoperative sore throat often employed a four-level classification system that categorized the presence and severity of postoperative sore throat as none, mild, moderate, or severe. When a study used this classification system, we considered the incidence of sore throat as the sum of mild, moderate, and severe cases.

Our secondary outcomes included 1) severity of postoperative sore throat, 2) incidence of moderate or severe sore throat, 3) presence of cough, 4) presence of hoarseness at $24 \mathrm{hr}$ after surgery/extubation, and 5) occurrence of adverse events. We accepted any scales that indicated the severity of postoperative sore throat. Because there is no universal definition for postoperative cough or hoarseness, we accepted the definition used in each study. Nevertheless, studies related to postoperative sore throat occasionally use a four-level classification system to rate cough or hoarseness as none, mild, moderate, and severe. For these studies, we calculated the incidence of cough or hoarseness from the sum of mild, moderate, and severe cases. We accepted the definition of adverse events from each study.
Search strategy

We searched Medline, EMBASE, China National Knowledge Infrastructure, and the Cochrane Central Register of Controlled Trials for eligible studies. Additionally, we searched Google Scholar and ClinicalTrials.gov to identify unpublished studies and reviewed the reference lists of the resulting publications. We imposed no restrictions on publication status or language. Our search strategy is provided in Table 1 . We updated the search on 6 October, 2018.

Study selection

The first author (A.K.) with one of the other authors (H.M. or R.S.) independently screened the title and abstracts obtained through the search to select eligible studies. There was no disagreement between the authors.

\section{Data extraction}

The same pair of authors independently extracted the following data from each study: patient characteristics (age, sex, American Society of Anesthesiologists [ASA] physical status), study characteristics (country, type of surgery, and length of surgery or anesthesia), interventions used (dose and form of magnesium as well as comparators), and outcomes of interest.

\section{Risk of bias assessment}

Independently and in duplicate, the same pair of authors that selected the studies and extracted the data assessed the risk of bias using the Cochrane risk of bias assessment tool. ${ }^{36}$ Because sore throat is a subjective outcome, we considered a study to have a low risk of performance bias when the investigators blinded participants to the intervention they received until all evaluations were completed. We also checked for conflicts of interest or industry sponsorship. We resolved any inconsistencies through discussion. If an e-mail address was available, we contacted the original study authors for details related to study methodology and unpublished outcome data. We considered the authors to be unresponsive if we obtained no reply after three contact attempts.

Statistical analysis

We calculated the risk ratio (RR) for dichotomous outcomes. Because all relevant studies used difference scales, we calculated and analyzed standardized mean difference (SMD) as a continuous variable for the severity of postoperative sore throat. We pooled the data into a 
Table 1 Search strategy

\begin{tabular}{|c|c|}
\hline 1. & 'magnesium'/exp OR 'magnesium’ \\
\hline 2. & 'magnesium sulfate'/exp OR 'magnesium sulfate' \\
\hline 3. & magnesium:ab,ti \\
\hline 4. & mgso4:ab,ti \\
\hline 5. & 'magnesium sulfate':ab,ti \\
\hline 6. & 1 OR 2 OR 3 OR 4 OR 5 \\
\hline 7. & ('pharyngitis'/exp OR 'pharyngitis' \\
\hline 8. & 'endotracheal intubation'/exp OR 'endotracheal intubation' \\
\hline 9. & (sore* OR inflamm* OR infect*) NEAR/5 throat \\
\hline 10. & pharyngit* OR ((endo*tracheal OR intra*tracheal) NEAR/5 intub*) \\
\hline 11. & 'postoperative sore throat'/exp OR 'postoperative sore throat' \\
\hline 12. & 7 OR 8 OR 9 OR 10 OR 11 \\
\hline 13. & 'randomized controlled trial'/exp \\
\hline 14. & 'randomised controlled trial'/exp \\
\hline 15. & 'randomization'/exp OR 'randomisation'/exp \\
\hline 16. & 'controlled study'/exp \\
\hline 17. & 'multicenter study'/exp \\
\hline 18. & 'phase 3 clinical trial'/exp \\
\hline 19. & 'phase 4 clinical trial'/exp \\
\hline 20. & 'double blind procedure'/exp \\
\hline 21. & 'single blind procedure'/exp \\
\hline 22. & random*:ti,ab \\
\hline 23. & cross*over:ti,ab \\
\hline 24. & factorial*:ti,ab \\
\hline 25. & placebo*:ti,ab \\
\hline 26. & volunteer*:ti,ab \\
\hline 27. & $(($ singl* OR doubl* OR trebl* OR tripl*) NEAR/5 (blind* OR mask*))) \\
\hline 28. & 13 OR 14 OR 15 OR 16 OR 17 OR 18 OR 19 OR 20 OR 21 OR 22 OR 23 OR 24 OR 25 OR 26 OR 27 \\
\hline 29. & 6 AND 12 AND 27 \\
\hline
\end{tabular}

single arm when a study examined different doses of magnesium. ${ }^{37}$ We added a continuity correction of 0.5 to each cell of the $2 \times 2$ table from the trial if a study included zero events in either arm. ${ }^{38}$ We pooled the data using the DerSimonian and Laird random-effects model. ${ }^{39} \mathrm{We}$ evaluated statistical heterogeneity using $Q$ and $I^{2}$ statistics $^{40}$ and considered an $I^{2} \geq 50 \%$ as showing large statistical heterogeneity. We conducted subgroup analyses to examine any differences in effect size for magnesium using a test of interaction between the type of applying magnesium. Because the number of studies for each outcome was less than ten, we did not test for publication bias according to Cochrane methodology. ${ }^{36}$

We conducted sensitivity analyses by excluding trials at unclear or high risk of bias in terms of sequence generation, allocation concealment, blinding of participants and outcome assessors, and conflicts of interest/industry sponsorship.

We conducted meta-regression analysis to examine the dose-response relationship for the incidence of postoperative sore throat. We anticipated that a larger preventive effect would be associated with a larger dose of magnesium. We converted the dose of magnesium to a scale of $\mathrm{mg}$ using the mean body weight reported in each respective study.

We conducted trial sequential analysis using the O'Brien-Fleming alpha-spending function to examine our findings' robustness against type 1 and type 2 errors. ${ }^{41,42}$ We applied a $5 \%$ risk of type 1 errors and a power of $80 \%$. Further, we obtained the required information size according to a relative risk reduction of $20 \%$ for postoperative sore throat, which is considered clinically conservative. $^{41}$ The threshold for statistical significance was set at $P<0.05$. We used Stata SE, version 15.1 (StataCorp., College Station, TX, USA) and TSA software, version 0.9 beta (Copenhagen Trial Unit, Copenhagen, Denmark) to perform conventional meta-analyses and to conduct the trial sequential analyses, respectively. 


\section{Results}

Overview of included studies

Our initial search yielded 364 titles and abstracts, and an additional search found seven articles. Eighteen studies were excluded after screening the full texts (eTable 1, available as Electronic Supplementary Material [ESM]). After application of our inclusion and exclusion criteria, 11 randomized-controlled trials involving 1,096 study participants were included in our analysis (Fig. 1). ${ }^{43-53}$

The mean participant ages in the included studies ranged from 25.4-56 yr, and the reported proportions of female participants ranged from $27-100 \%$ (Table 2). Nine trials included patients with an ASA status of I-II, ${ }^{43-46,48-51,53}$ while two trials included patients with an ASA status of IIII. $^{47,52}$ Nine trials presented surgery type, including thoracic surgery, orthopedic surgery, abdominal surgery (appendectomy, open cholecystectomy, laparoscopic cholecystectomy, or laparoscopic surgery of ovarian cyst), or surgery of the abdomen and lower limbs. ${ }^{43,45-48,50-53}$ All trials but one included elective surgery. The median sample size was 100 (range, 40-225).

Topical magnesium was applied as a single dose prior to surgery in all studies. Seven trials administered magnesium as nebulization, ${ }^{44-47,49,50,53}$ three administered it as gargle, ${ }^{48,51,52}$ and one administered it as lozenges. ${ }^{43}$ The dose of magnesium was fixed in six studies $(100 \mathrm{mg}$, one study $^{43} ; 225 \mathrm{mg}$, four studies ${ }^{45,46,49,53} ; 250 \mathrm{mg}$, one study ${ }^{47} ; 500 \mathrm{mg}$, one study ${ }^{47}$ ) and titrated according to patient body weight in three studies $\left(20 \mathrm{mg} \cdot \mathrm{kg}^{-1}\right){ }^{48,51,52}$ The remaining study did not report the dose. ${ }^{44} \mathrm{~A}$ placebo was used in one study as the control, ${ }^{43}$ while topical saline was used as a non-analgesic control in eight studies, ${ }^{45-47,49-53}$ two ${ }^{51,52}$ of which used dextrose water as a solvent. Active controls (agents with known analgesic effects) were used in six studies, including ketamine (gargle, two studies ${ }^{48,51}$; nebulization, two studies), ${ }^{46,47}$ aerosolized dexamethasone (one study), ${ }^{44}$ and lidocaine applied over endotracheal tubes (one study). ${ }^{52}$ All studies but one administered magnesium 15 to $30 \mathrm{~min}$ before surgery or anesthesia induction, while the remaining study administered nebulized magnesium $30 \mathrm{sec}$ before intubation. $^{50}$ Six studies were conducted in India, ${ }^{44-47,49,53}$ three in China, ${ }^{50-52}$ and one each in $\operatorname{Iran}^{48}$ and Turkey. ${ }^{43}$ All studies were published in full between 2012 and 2018. Seven studies ${ }^{43-49,53}$ were reported in English, and the remaining three were reported in Chinese. ${ }^{50-52}$

Four studies used a four-level classification system to rate the presence and severity of postoperative sore throat, ${ }^{43,46,47,53}$ while the other seven reported only the incidence of postoperative sore throat. Four studies used a visual analogue scale (VAS) to rate severity. ${ }^{48,50-52}$ One study used a VAS ranging from 0 to $100,{ }^{50}$ one used a VAS ranging from 0 to ${ }^{5,48}$ and the other two did not report the details of the VAS used. ${ }^{51,52}$ We contacted nine study

Fig. 1 PRISMA flowchart

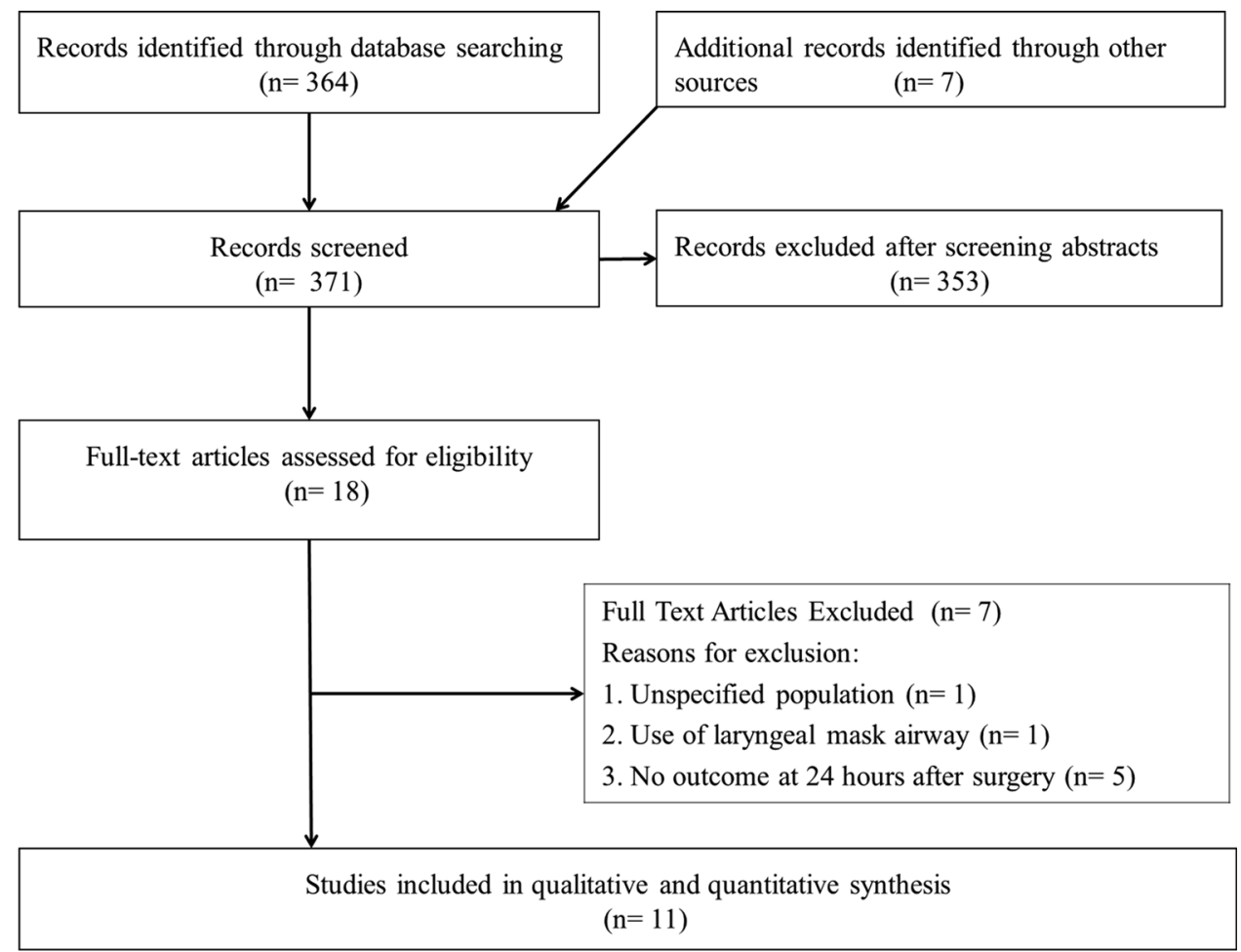


Table 2 Characteristics of included studies

\begin{tabular}{|c|c|c|c|c|c|c|c|c|c|c|}
\hline Study/year & Country & $\begin{array}{l}\text { Sample } \\
\text { size }(\% \\
\text { female })\end{array}$ & $\begin{array}{l}\text { Mean } \\
\text { age }\end{array}$ & $\begin{array}{l}\text { ASA- } \\
\text { PS }\end{array}$ & Surgery & $\begin{array}{l}\text { ETT } \\
\text { size } \\
(\mathrm{mm})\end{array}$ & $\begin{array}{l}\text { Cuff } \\
\text { pressure } \\
\left(\mathrm{cmH}_{2} \mathrm{O}\right)\end{array}$ & Intervention & $\begin{array}{l}\text { Duration } \\
\text { (minutes) }\end{array}$ & $\begin{array}{l}\text { Postoperative } \\
\text { sore throat } \\
\text { measurement } \\
\text { at } 24 \mathrm{hr} \text { after } \\
\text { surgery }\end{array}$ \\
\hline $\begin{array}{c}\text { Borazan/ } \\
2012\end{array}$ & Turkey & $70(30)$ & 39.5 & I, II & $\begin{array}{l}\text { Elective } \\
\text { orthopedic } \\
\text { surgery of lower } \\
\text { extremities }\end{array}$ & $\begin{array}{l}8.5 \\
(\mathrm{~m}) \\
7.5(\mathrm{f})\end{array}$ & $20-22$ & $\begin{array}{l}\text { 1. Mg lozenges ( } 100 \\
\mathrm{mg}) \\
\text { 2. Placebo given } 30 \mathrm{~min} \\
\text { before surgery }\end{array}$ & $\begin{array}{l}\text { Surgery: } \\
\quad 80.5 \\
\text { Anesthesia: } \\
\quad 85\end{array}$ & $\begin{array}{l}\text { 4-level scoring } \\
\text { system }\end{array}$ \\
\hline Gupta/2012 & India & 40 (NR) & NR & I, II & $\begin{array}{l}\text { Elective open } \\
\text { cholecystectomy }\end{array}$ & NR & NR & $\begin{array}{l}\text { 1. Nebulized } \mathrm{MgSO}_{4} \\
(225 \mathrm{mg}) \\
\text { 2. Nebulized saline } \\
\text { Initiated } 20 \text { min (ending } \\
15 \mathrm{~min} \text { ) before } \\
\text { anesthesia induction }\end{array}$ & NR & $\begin{array}{l}\text { No. of } \\
\text { incidence }\end{array}$ \\
\hline $\mathrm{Wu} / 2013$ & China & $48(54)$ & 56 & I, II & $\begin{array}{l}\text { Elective thoracic } \\
\text { surgery }\end{array}$ & DLT & NR & $\begin{array}{l}\text { 1. Sprayed MgSO4 (500 } \\
\text { mg) on throat } \\
\text { 2. Sprayed saline } 2 \mathrm{~mL} \\
\text { on throat given } 30 \\
\text { sec before tracheal } \\
\text { intubation }\end{array}$ & $\begin{array}{l}\text { Anesthesia: } \\
219.3\end{array}$ & $\begin{array}{l}\text { No. of } \\
\quad \text { incidence } \\
\text { VAS }\end{array}$ \\
\hline $\begin{array}{l}\text { Teymourian/ } \\
2015\end{array}$ & Iran & $\begin{array}{l}100 \\
(54)\end{array}$ & 30 & I, II & $\begin{array}{l}\text { Emergency } \\
\text { surgery of acute } \\
\text { appendicitis }\end{array}$ & NR & $20-30$ & $\begin{array}{l}\text { 1. Gargle } \mathrm{MgSO}_{4}(20 \\
\left.\mathrm{mg} \cdot \mathrm{kg}^{-1}\right) \text { in } 20 \% \\
\text { dextrose water } \\
\text { 2. Gargle ketamine }(0.5 \\
\left.\mathrm{mg} \cdot \mathrm{kg}^{-1}\right) \text { in } 20 \% \\
\text { dextrose water given } \\
15 \text { min before } \\
\text { surgery }\end{array}$ & $\begin{array}{l}\text { Anesthesia: } \\
54.2\end{array}$ & $\begin{array}{l}\text { No. of } \\
\quad \text { incidence } \\
\text { VAS }\end{array}$ \\
\hline Lin/2016 & China & $\begin{array}{l}115 \\
(100)\end{array}$ & 42.7 & I-III & $\begin{array}{l}\text { Laparoscopic } \\
\text { surgery of } \\
\text { ovarian cyst }\end{array}$ & 7 & NR & $\begin{array}{l}\text { 1. Gargle } \mathrm{MgSO}_{4}(20 \\
\left.\mathrm{mg} \cdot \mathrm{kg}^{-1}\right) 5 \% \\
\text { dextrose water of } 30 \\
\mathrm{~mL} \\
\text { 2. Lidocaine applied on } \\
\text { one-third of the ETT } \\
\text { 3. Saline } 2 \mathrm{~mL} \text { in } 5 \% \\
\text { dextrose water of } 30 \\
\text { mL given } 15 \mathrm{~min} \\
\text { before surgery }\end{array}$ & $\begin{array}{l}\text { Surgery: } \\
\quad 71.2 \\
\text { Anesthesia: } \\
\quad 91.6\end{array}$ & $\begin{array}{l}\text { No. of } \\
\quad \text { incidence } \\
\text { VAS }\end{array}$ \\
\hline Yadav/2016 & India & $\begin{array}{l}100 \\
(47)\end{array}$ & 40.9 & I, II & Elective surgery & $\begin{array}{l}8(\mathrm{~m}) \\
7(\mathrm{f})\end{array}$ & 20 & $\begin{array}{l}\text { 1. Nebulized } \mathrm{MgSO}_{4} \\
(225 \mathrm{mg}) \\
\text { 2. Saline } 3 \mathrm{~mL} \\
\text { Initiated } 15 \mathrm{~min} \text { (ending } \\
5 \mathrm{~min} \text { ) before } \\
\text { anesthesia induction }\end{array}$ & NR & $\begin{array}{l}\text { No. of } \\
\text { incidence }\end{array}$ \\
\hline Jain/2017 & India & $\begin{array}{l}225 \\
(100)\end{array}$ & NR & I, II & $\begin{array}{l}\text { Elective } \\
\text { laparoscopic } \\
\text { cholecystectomy }\end{array}$ & $7-7.5$ & 20 & $\begin{array}{l}\text { 1. Nebulized } \mathrm{MgSO}_{4} \\
(225 \mathrm{mg}) \\
\text { 2. Nebulized ketamine } \\
(50 \mathrm{mg}) \\
\text { 3. Nebulized saline } 3 \\
\text { mL initiated } 15 \mathrm{~min} \\
\text { (ending } 5 \text { min) before } \\
\text { anesthesia induction }\end{array}$ & NR & $\begin{array}{l}\text { 4-level scoring } \\
\text { system }\end{array}$ \\
\hline
\end{tabular}


Table 2 continued

\begin{tabular}{|c|c|c|c|c|c|c|c|c|c|c|}
\hline Study/year & Country & $\begin{array}{l}\text { Sample } \\
\text { size }(\% \\
\text { female })\end{array}$ & $\begin{array}{l}\text { Mean } \\
\text { age }\end{array}$ & $\begin{array}{l}\text { ASA- } \\
\text { PS }\end{array}$ & Surgery & $\begin{array}{l}\text { ETT } \\
\text { size } \\
(\mathrm{mm})\end{array}$ & $\begin{array}{l}\text { Cuff } \\
\text { pressure } \\
\left(\mathrm{cmH}_{2} \mathrm{O}\right)\end{array}$ & Intervention & $\begin{array}{l}\text { Duration } \\
\text { (minutes) }\end{array}$ & $\begin{array}{l}\text { Postoperative } \\
\text { sore throat } \\
\text { measurement } \\
\text { at } 24 \mathrm{hr} \text { after } \\
\text { surgery }\end{array}$ \\
\hline Rajan/2017 & India & 60 (NR) & NR & I-III & $\begin{array}{l}\text { Elective surgery of } \\
\text { abdomen and } \\
\text { lower limbs }\end{array}$ & $\begin{array}{r}8-8.5 \\
(\mathrm{~m}) \\
7-7.5 \\
(\mathrm{f})\end{array}$ & $20-25$ & $\begin{array}{l}\text { 1. Nebulized } \mathrm{MgSO}_{4} \\
250 \mathrm{mg} \text { in saline } 5 \\
\mathrm{~mL} \\
\text { 2. Nebulized } \mathrm{MgSO}_{4} \\
500 \mathrm{mg} \text { in saline } 5 \\
\mathrm{~mL} \\
\text { 3. Nebulized ketamine } \\
50 \mathrm{mg} \text { in saline } 5 \mathrm{~mL} \\
\text { 4. Saline given } 15 \mathrm{~min} \\
\text { before surgery }\end{array}$ & NR & $\begin{array}{l}\text { 4-level scoring } \\
\text { system }\end{array}$ \\
\hline $\begin{array}{c}\text { Sharma/ } \\
2017\end{array}$ & India & $\begin{array}{l}140 \\
(30)\end{array}$ & 38.9 & I, II & $\begin{array}{l}\text { Lumbar spine } \\
\text { surgery }\end{array}$ & $\begin{array}{l}8-8.5 \\
(\mathrm{~m}) \\
7-7.5 \\
(\mathrm{f})\end{array}$ & 20 & $\begin{array}{l}\text { 1. Nebulized } \mathrm{MgSO}_{4} \\
225 \mathrm{mg} \text { in saline } 5 \\
\mathrm{~mL} \\
\text { 2. Saline initiated } 20 \\
\text { min (ending 5) before } \\
\text { anesthesia induction }\end{array}$ & $\begin{array}{c}\text { Surgery: } \\
129.6\end{array}$ & $\begin{array}{l}\text { 4-level scoring } \\
\text { system }\end{array}$ \\
\hline $\begin{array}{l}\text { Ashwini/ } \\
2018\end{array}$ & India & $80(36)$ & 37.3 & I, II & Elective surgery & $\begin{array}{l}8(\mathrm{~m}) \\
7(\mathrm{f})\end{array}$ & $20-22$ & $\begin{array}{l}\text { 1. Nebulized } \mathrm{MgSO}_{4} \\
\text { 2. Nebulized } \\
\text { dexamethasone } 8 \mathrm{mg} \\
\text { initiated } 30 \mathrm{~min} \\
\text { before anesthesia } \\
\text { induction }\end{array}$ & $\begin{array}{c}\text { Surgery: } \\
116.4\end{array}$ & $\begin{array}{l}\text { No. of } \\
\quad \text { incidence }\end{array}$ \\
\hline Shen/2018 & China & $\begin{array}{l}118 \\
(27)\end{array}$ & 25.4 & I, II & $\begin{array}{l}\text { Elective } \\
\text { thoracoscopic } \\
\text { resection of } \\
\text { bullae }\end{array}$ & DLT & NR & $\begin{array}{l}\text { 1. Gargle } \mathrm{MgSO}_{4}(20 \\
\mathrm{mg} / \mathrm{kg}) \text { in } 5 \% \\
\text { dextrose water } 30 \mathrm{~mL} \\
\text { 2. Gargle ketamine }(0.5 \\
\left.\mathrm{mg} \cdot \mathrm{kg}^{-1}\right) \text { in } 5 \% \\
\text { dextrose water } 30 \mathrm{~mL} \\
\text { 3. Gargle saline }(2 \mathrm{~mL}) \\
\text { in } 5 \% \text { dextrose water } \\
30 \mathrm{~mL} \text { initiated } 15 \\
\text { min before anesthesia } \\
\text { induction }\end{array}$ & $\begin{array}{l}\text { Surgery: } \\
65.2\end{array}$ & $\begin{array}{l}\text { No. of } \\
\text { incidence } \\
\text { VAS }\end{array}$ \\
\hline
\end{tabular}

ASA-PS = American Society of Anesthesiologists-physical status; DLT = double-lumen tube; ETT = endotracheal tube; $\mathrm{f}=$ female; $\mathrm{m}=$ male; $\mathrm{Mg}=$ magnesium $; \mathrm{MgSO}_{4}=$ magnesium sulfate; $\mathrm{NR}=$ not reported; $\mathrm{VAS}=$ visual analogue scale

authors for further information, and three responded with information.

\section{Risk of bias}

Seven of the ten studies ultimately included in this analysis conducted adequate sequence generation, while six of the studies performed adequate allocation concealment (Table 3). Participants and outcome assessors were adequately blinded in three and six studies, respectively. Four studies were free from conflicts of interest or sponsorship. Two studies showed a high risk of selective reporting outcome bias: one of these embedded the outcomes in figures, ${ }^{48}$ and the other reported only an interpretation of the results. ${ }^{45}$ Consequently, we narratively reviewed one study ${ }^{45}$ and conducted meta-analysis with data available from the other studies.

Magnesium vs non-analgesic controls

\section{Incidence of postoperative sore throat}

Eight studies with a total of 659 participants provided data on the incidence of sore throat at $24 \mathrm{hr}$ after surgery/ extubation. ${ }^{43,46,47,49-53}$ Topical application of magnesium was associated with a lower incidence of postoperative sore throat compared with non-analgesic controls (27/336 [8\%] vs $93 / 323$ [29\%], respectively; RR, 0.31 ; $95 \%$ confidence interval [CI], 0.21 to $0.45 ; P<0.001 ; I^{2}=0.0 \%$; Fig. 2; risk difference, $-0.18 ; 95 \% \mathrm{CI}, 0.23$ to $0.13 ; P<0.001 ; I^{2}$ 
Table 3 Risk of bias in included studies

\begin{tabular}{|c|c|c|c|c|c|c|c|c|}
\hline Study & $\begin{array}{l}\text { Sequence } \\
\text { generation }\end{array}$ & $\begin{array}{l}\text { Allocation } \\
\text { concealment }\end{array}$ & $\begin{array}{l}\text { Blinding of } \\
\text { participants and } \\
\text { personnel }\end{array}$ & $\begin{array}{l}\text { Blinding of } \\
\text { outcome } \\
\text { assessors }\end{array}$ & $\begin{array}{l}\text { Incomplete } \\
\text { outcome } \\
\text { data }\end{array}$ & $\begin{array}{l}\text { Selective } \\
\text { outcome } \\
\text { reporting }\end{array}$ & $\begin{array}{l}\text { Other } \\
\text { source of } \\
\text { bias }\end{array}$ & $\begin{array}{l}\text { Industry } \\
\text { sponsorship/ conflict } \\
\text { of interest }\end{array}$ \\
\hline $\begin{array}{l}\text { Borazan/ } \\
2012\end{array}$ & Low & Low & Low & Low & Low & Low & Low & None \\
\hline Gupta/2012 & Unclear & Unclear & Unclear & Unclear & Unclear & High & Unclear & Unclear \\
\hline $\mathrm{Wu} / 2013$ & Unclear & Unclear & Unclear & Unclear & Low & Low & Low & Unclear \\
\hline $\begin{array}{l}\text { Teymourian/ } \\
2015\end{array}$ & Low & Low & Low & Low & Low & High & Low & None \\
\hline $\operatorname{Lin} / 2016$ & Low & Unclear & Unclear & Unclear & Low & Low & Low & Unclear \\
\hline Yadav/2016 & Low & Low & Unclear & Low & Unclear & Low & Unclear & Unclear \\
\hline Jain/2017 & Low & Low & Unclear & Unclear & Low & Low & Low & None \\
\hline Rajan/2017 & Low & Unclear & Low & Low & Low & Low & Unclear & None \\
\hline $\begin{array}{c}\text { Sharma/ } \\
2018\end{array}$ & Unclear & Low & Unclear & Low & Low & Low & Low & Unclear \\
\hline $\begin{array}{l}\text { Ashwini/ } \\
2018\end{array}$ & Unclear & Low & Unclear & Low & Low & Low & Low & Unclear \\
\hline Shen/2018 & Low & Unclear & Unclear & Unclear & Low & Low & Low & Unclear \\
\hline
\end{tabular}

Fig. 2 Forest plot on the incidence of postoperative sore throat at $24 \mathrm{hr}$ ( $v s$ non-analgesic controls). $\mathrm{RR}=$ risk ratio

\begin{tabular}{|c|c|c|c|c|c|}
\hline \multirow{2}{*}{\multicolumn{2}{|c|}{ Trial (Year) }} & \multirow[b]{2}{*}{$\operatorname{RR}(95 \% \mathrm{CI})$} & \multicolumn{2}{|c|}{ Events/ Total } & \multirow{2}{*}{$\begin{array}{l}\text { Weight } \\
(\%)\end{array}$} \\
\hline & & & Magnesium & Control & \\
\hline Borazan & 2012 & $0.30(0.09,1.00)$ & $3 / 35$ & $10 / 35$ & 10.41 \\
\hline Wu & 2013 & $0.30(0.09,0.96)$ & $3 / 24$ & $10 / 24$ & 11.19 \\
\hline Lin & 2016 & $0.36(0.16,0.82)$ & $6 / 38$ & $17 / 39$ & 22.58 \\
\hline Yadav & 2016 & $0.07(0.00,1.14)$ & $0 / 50$ & $7 / 50$ & 1.87 \\
\hline Jain & 2017 & $0.29(0.06,1.31)$ & $2 / 50$ & $7 / 50$ & 6.50 \\
\hline Sharma & 2017 & $0.40(0.19,0.85)$ & $7 / 39$ & $18 / 40$ & 26.51 \\
\hline Rajan & 2018 & $0.08(0.01,0.63)$ & $1 / 30$ & $6 / 15$ & 3.67 \\
\hline Shen & 2018 & $0.28(0.11,0.71)$ & $5 / 70$ & $18 / 70$ & 17.26 \\
\hline \multicolumn{2}{|c|}{ Overall $($ I-squared $=0.0 \%$ ) } & $0.31(0.21,0.45)$ & $27 / 336$ & $93 / 323$ & 100.00 \\
\hline \multicolumn{6}{|c|}{ Note: Weights are from random effects analysis. } \\
\hline & 0.004 & 256 & & & \\
\hline
\end{tabular}

$=0.0 \%$. One study narratively concluded that topical magnesium reduced the incidence of sore throat. ${ }^{45}$

Severity of postoperative sore throat

Fig. 3). One study narratively concluded that topical magnesium reduced the severity of sore throat. ${ }^{45}$

Moderate or severe postoperative sore throat

Three studies including a total of 254 participants provided data on the severity of sore throat at $24 \mathrm{hr}$ after surgery/ extubation. $^{50-52}$ Topical application of magnesium was associated with a lower severity score for postoperative sore throat compared with non-analgesic controls (SMD, $2.66 ; 95 \% \mathrm{CI},-3.89$ to $-1.43 ; P<0.001 ; I^{2}=90.1 \%$;
Four studies with a total of 356 participants provided data on the incidence of moderate or severe sore throat at $24 \mathrm{hr}$ after surgery/extubation. ${ }^{43,46,47,53}$ Topical application of magnesium was associated with a reduced incidence of moderate or severe postoperative sore throat compared with non-analgesic controls (1/185 vs 18/171, respectively; 
Fig. 3 Forest plot on the severity of postoperative sore throat at $24 \mathrm{hr}$ ( $v s$ non-analgesic controls). SMD = standardized mean difference

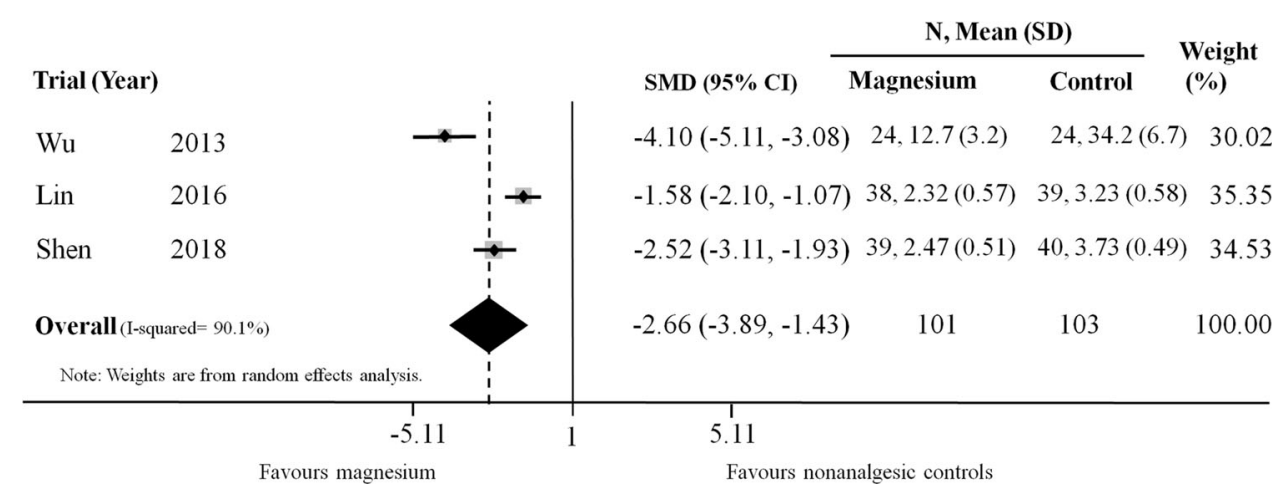

Fig. 4 Forest plot on the incidence of moderate or severe postoperative sore throat at 24 $\mathrm{hr}$ ( $v s$ non-analgesic controls). $\mathrm{RR}=$ risk ratio

\begin{tabular}{|c|c|c|c|c|c|}
\hline \multirow{2}{*}{\multicolumn{2}{|c|}{ Trial (Year) }} & \multirow[b]{2}{*}{$\mathrm{RR}(95 \% \mathrm{CI})$} & \multicolumn{2}{|c|}{ Events/ Total } & \multirow{2}{*}{$\begin{array}{l}\text { Weight } \\
(\%)\end{array}$} \\
\hline & & & Magnesium & Control & \\
\hline Borazan & 2012 & $0.11(0.01,1.99)$ & $0 / 35$ & $4 / 35$ & 20.71 \\
\hline Jain & 2017 & $0.14(0.01,2.70)$ & $0 / 50$ & $3 / 50$ & 19.98 \\
\hline Sharma & 2017 & $0.12(0.03,0.45)$ & $1 / 70$ & $10 / 70$ & 41.89 \\
\hline Rajan & 2018 & $0.10(0.01,0.76)$ & $0 / 30$ & $1 / 16$ & \\
\hline \multirow{2}{*}{\multicolumn{2}{|c|}{$\begin{array}{l}\text { Overall (I-squared }=0.0 \%) \\
\text { Note: Weights are from random effects analysis. }\end{array}$}} & $0.12(0.03,0.45)$ & $1 / 185$ & $18 / 171$ & 100.00 \\
\hline & & & & & \\
\hline & 0.06 & $\begin{array}{c}1 \\
161\end{array}$ & & & \\
\hline
\end{tabular}

RR, $0.12 ; 95 \%$ CI, 0.03 to $0.45 ; P=0.002 ; I^{2}=0.0 \%$; Fig. 4).

Postoperative cough

Only one study involving 45 participants provided data on the incidence of cough at $24 \mathrm{hr}$ after surgery/extubation. ${ }^{47}$ There was no incidence of postoperative cough in any study group (control, $\mathrm{MgSO}_{4} 250 \mathrm{mg}$ or $500 \mathrm{mg}$ ) at $24 \mathrm{hr}$ after surgery/extubation.

\section{Postoperative hoarseness}

Three studies including 216 participants provided data on the incidence of hoarseness at $24 \mathrm{hr}$ after surgery/ extubation. ${ }^{43,46,47}$ Topical administration of magnesium was associated with a reduced incidence of postoperative hoarseness compared with non-analgesic controls $(0 / 115$ [0\%] vs 8/101 [8\%], respectively; RR, 0.14; 95\% CI, 0.03 to $0.79 ; P=0.026 ; I^{2}=0.0 \%$; eFig. 1 , available as ESM).

\section{Adverse events}

Only three of the ten studies included information on adverse events. One study reported that there were no adverse events in either the magnesium or the control group $^{53}$ and the other two disclosed that there were no systemic or local adverse events associated with topical magnesium. ${ }^{43,49}$

Subgroup, sensitivity, and trial sequential analyses

There were no significant differences in the incidence of overall or moderate/severe postoperative sore throat between subgroups by method of magnesium application $(P=0.63$ and $P=0.95$ based on a test of interaction, respectively; eTable 2, available as ESM). Nevertheless, nebulized magnesium showed a larger preventive effect regarding sore throat severity compared with magnesium gargle $(P=0.004)$. Most sensitivity analyses for severity of postoperative sore throat were impossible because studies with a low risk of bias were lacking; nevertheless, the results of the other sensitivity analyses were mostly consistent with their respective primary analyses (eTable 2, available as ESM). The results of the trial sequential analysis showed that the cumulative z-curve crossed both the conventional and trial sequential monitoring boundaries for benefit before reaching the required information size (1,747 participants), which supports the positive efficacy of topical magnesium in preventing postoperative sore throat (eFig. 2, available as ESM).

We examined the relationship between the effect size and the dose of topical magnesium using meta-regression. 
We calculated the dose of topical magnesium in two studies $^{51,52}$ based on the mean body weight reported in each study. Nevertheless, no dose-response relationship was detected ( $P=0.79$; eFig. 3, available as ESM).

\section{Magnesium vs active controls}

Six trials employed analgesic agents as comparators. These included ketamine (gargle, two studies ${ }^{48,51}$; nebulization, two studies, ${ }^{46,47}$ aerosolized dexamethasone (one study), ${ }^{44}$ and lidocaine applied over endotracheal tubes (one study). ${ }^{52}$

With regard to the prevention of postoperative sore throat, topical administration of magnesium was superior to lubrication of the endotracheal tube with lidocaine (RR, $0.35 ; 95 \% \mathrm{CI}, 0.16$ to 0.82 ) and topical ketamine (RR, $0.54 ; 95 \% \mathrm{CI}, 0.34$ to 0.86 ) but was similar to aerosolized corticosteroids (RR, 1.02; 95\% CI, 0.07 to 15.83) (eTable 3, available as ESM). For severity of postoperative sore throat, topical magnesium performed better than topical lidocaine but was statistically similar to topical ketamine. For incidence of hoarseness, topical administration of magnesium was statistically similar to topical lidocaine or ketamine.

\section{Discussion}

Our study suggests that, compared with non-analgesic controls, the topical application of magnesium attenuates the incidence and severity of postoperative sore throat in adults undergoing tracheal intubation for surgery under general anesthesia. Our analysis also suggests that the number of patients needed to prevent one incidence of postoperative sore throat with topical magnesium is five (95\% CI, 4 to 8), signifying that topical magnesium provides a large prophylactic benefit. Topical application of magnesium was also observed to decrease the incidence of moderate or severe sore throat and hoarseness after surgery, though its efficacy in preventing cough is still uncertain. The limited available evidence suggests that topical administration of magnesium is not associated with significant adverse events. Our primary findings were mostly robust according to the sensitivity analyses, except for those regarding severity of sore throat. Our trial sequential analysis suggests that the evidence on the efficacy of topical magnesium in preventing postoperative sore throat is adequate.

Mucosal inflammation around the tracheal tube cuff is considered the likely etiology predisposing sore throat following extubation. ${ }^{10}$ Previous studies suggest that antiinflammatory agents, such as topical benzydamine hydrochloride, topical licorice, intravenous dexamethasone, and topical betamethasone and dexamethasone, prevent postoperative sore throat in surgical patients. ${ }^{13-18}$ Intravenous corticosteroids administered before elective extubation have been shown to prevent laryngeal edema and post-extubation airway complications in critically ill patients. ${ }^{54}$ Consistent with these previous findings, the results of the current study suggest that the anti-inflammatory effect of topical administration of magnesium is associated with a reduced risk of postoperative sore throat.

There was substantial statistical heterogeneity in the pooled data regarding severity of postoperative sore throat, whereas no statistical heterogeneity was found for the other outcomes. The main reason for this discrepancy was probably the clinical heterogeneity across the three studies that were pooled for the analysis of severity. Two studies used gargle, ${ }^{51,52}$ while the other study used nebulization. ${ }^{50}$ Further, the two studies that used gargle each used different sizes of endotracheal tubes: one used 7-mm diameter single-lumen tubes ${ }^{52}$ and the other used double-lumen tubes. ${ }^{51}$ Although intubation with double-lumen tubes is known to increase the severity of postoperative sore throat, ${ }^{55}$ the study that used double-lumen tubes ${ }^{51}$ showed a larger preventive effect than the study that used single-lumen tubes, ${ }^{52}$ which is beyond our explanation. Given the large clinical heterogeneity among studies for this outcome, our findings regarding sore throat severity should be interpreted cautiously.

Our meta-regression analysis was unable to find an association between dose of topical magnesium and effect size, possibly because the point estimates of the efficacy in most trials were similar. Our study thus could not elucidate the optimal dose of magnesium for preventing postoperative sore throat, but all doses used in the included studies are likely sufficient to prevent postoperative sore throat.

Previous studies have shown that topical application of ketamine ${ }^{56}$ lidocaine,${ }^{57}$ and aerosolized corticosteroids ${ }^{15}$ prevent postoperative sore throat. Our study suggests that topical magnesium might be superior to topical ketamine and lidocaine but similar to topical corticosteroids. Nevertheless, comparisons of the effectiveness of these treatments were performed in only a low number of small studies. Studies examining the comparative effectiveness of topical magnesium $v s$ other pharmacologic options in preventing postoperative sore throat are still warranted.

Our review fails to sufficiently assess adverse events related to topical magnesium. The Consolidated Standards of Reporting Trials statement recommends that randomized-controlled trials should report all important harms or unintended effects in each study group. ${ }^{58}$ Although all of the studies included in our review were published after the announcement of this statement, only 
three reported on adverse events, and these gave only minimal details. Therefore, the safety of topical magnesium needs to be determined in further studies.

Our study has several key strengths. First, this is the first systematic review on the efficacy and safety of applying topical magnesium in preventing postoperative sore throat. Second, our comprehensive literature search found 11 studies, allowing us to perform sensitivity analyses for most outcomes. Third, we compared topical magnesium with agents that are known to prevent postoperative sore throat.

Our review is not without limitations. First, the number of pooled studies for each outcome was small despite our exhaustive search and attempts to gain unpublished outcomes. Second, there is a possibility of publication bias for each outcome. In line with the recommendations of the Cochrane Collaboration, we did not test for publication bias because the number of studies available for each outcome was less than ten. ${ }^{36}$ Third, we could not control for several potential confounding factors within the included trials. In particular, cuff pressure, tracheal tube size, and duration of intubation or surgery are known risk factors for postoperative sore throat. ${ }^{59}$ Nevertheless, these outcomes were inconsistently reported in the included studies, precluding our ability to adjust for these variables in our analysis. Fourth, two of the studies included in the meta-regression analysis determined magnesium dose based on patient body weight. We estimated the dose using the mean body weight reported in each study. This estimation might not always be correct because body weight and the subsequent efficacy of topical magnesium can vary according to sex. Fifth, our study considered both single-lumen and double-lumen tubes. Nevertheless, the efficacy of topical magnesium was obvious regardless of the type of endotracheal tube. Finally, topical magnesium is not part of clinical practice in North American countries, although it has been used and investigated in many Asian nations. Thus, the merits of topical magnesium in preventing postoperative sore throat needs to be assessed in North American populations.

In conclusion, our study confirms that preoperative topical administration of magnesium prevents postoperative sore throat more effectively than nonanalgesic strategies. Further studies are warranted to determine the optimal dose and safety of topical magnesium to prevent postoperative sore throat.

\section{Conflicts of interest None declared.}

Editorial responsibility This submission was handled by Dr. Philip M. Jones, Associate Editor, Canadian Journal of Anesthesia.

Author contributions Akira Kuriyama contributed to all aspects of this study, including study conception and design; acquisition, analysis, and interpretation of data; and drafting the article. Hirokazu Maeda and Rao Sun contributed to the acquisition and analysis of data and revision of the article.

Financial disclosures None.

\section{References}

1. Ahmed A, Abbasi S, Ghafoor HB, Ishaq M. Postoperative sore throat after elective surgical procedures. J Ayub Med Coll Abbottabad 2007; 19: 12-4.

2. Biro P, Seifert B, Pasch T. Complaints of sore throat after tracheal intubation: a prospective evaluation. Eur J Anaesthesiol 2005; 22: 307-11.

3. Christensen AM, Willemoes-Larsen H, Lundby L, Jakobsen KB. Postoperative throat complaints after tracheal intubation. $\mathrm{Br} \mathrm{J}$ Anaesth 1994; 73: 786-7.

4. Higgins PP, Chung F, Mezei G. Postoperative sore throat after ambulatory surgery. Br J Anaesth 2002; 88: 582-4.

5. Lehmann M, Monte $\mathrm{K}$, Barach $\mathrm{P}$, Kindler $\mathrm{CH}$. Postoperative patient complaints: a prospective interview study of 12,276 patients. J Clin Anesth 2010; 22: 13-21.

6. Maruyama K, Sakai H, Miyazawa $H$, et al. Sore throat and hoarseness after total intravenous anaesthesia. Br J Anaesth 2004; 92: 541-3.

7. Inoue $S$, Abe R, Tanaka $Y$, Kawaguchi $M$. Tracheal intubation by trainees does not alter the incidence or duration of postoperative sore throat and hoarseness: a teaching hospital-based propensity score analysis. Br J Anaesth 2015; 115: 463-9.

8. Macario A, Weinger M, Carney S, Kim A. Which clinical anesthesia outcomes are important to avoid? The perspective of patients. Anesth Analg 1999; 89: 652-8.

9. Chandler $M$. Tracheal intubation and sore throat: a mechanical explanation. Anaesthesia 2002; 57: 155-61.

10. Combes $X$, Schauvliege $F$, Peyrouset $O$, et al. Intracuff pressure and tracheal morbidity: influence of filling with saline during nitrous oxide anesthesia. Anesthesiology 2001; 95: 1120-4.

11. Donnelly WH. Histopathology of endotracheal intubation. An autopsy study of 99 cases. Arch Pathol 1969; 88: 511-20.

12. Loeser EA, Hodges M, Gliedman J, Stanley TH, Johansen RK, Yonetani D. Tracheal pathology following short-term intubation with low- and high-pressure endotracheal tube cuffs. Anesth Analg 1978; 57: 577-9.

13. Way WL, Sooy FA. Histologic changes produced by endotracheal intubation. Ann Otol Rhinol Laryngol 1965; 74: 799-812.

14. Kuriyama A, Maeda $H$. Topical application of licorice for prevention of postoperative sore throat in adults: a systematic review and meta-analysis. J Clin Anesth 2018; 54: 25-32.

15. Kuriyama A, Maeda H, Sun R, Aga M. Topical application of corticosteroids to tracheal tubes to prevent postoperative sore throat in adults undergoing tracheal intubation: a systematic review and meta-analysis. Anaesthesia 2018; 73: 1546-56.

16. Kuriyama $A$, Maeda $H$. Preoperative intravenous dexamethasone prevents tracheal intubation-related sore throat in adult surgical patients: a systematic review and meta-analysis. Can J Anesth 2019; DOI: 10.1007/s12630-018-01288-2.

17. Kuriyama A, Aga M, Maeda $H$. Topical benzydamine hydrochloride for prevention of postoperative sore throat in adults undergoing tracheal intubation for elective surgery: a systematic review and meta-analysis. Anaesthesia 2018; 73: 889900 . 
18. Kuriyama A, Maeda $H$, Sun $R$. Aerosolized corticosteroids to prevent postoperative sore throat in adults: a systematic review and meta-analysis. Acta Anaesthesiol Scand 2019; 63: 282-91.

19. Rodriguez-Rubio L, Nava E, Del Pozo JS, Jordan J. Influence of the perioperative administration of magnesium sulfate on the total dose of anesthetics during general anesthesia. A systematic review and meta-analysis. J Clin Anesth 2017; 39: 129-38.

20. Pascual-Ramirez, J, Gil-Trujillo S, Alcantarilla C. Intrathecal magnesium as analgesic adjuvant for spinal anesthesia: a metaanalysis of randomized trials. Minerva Anestesiol 2013; 79: 66778.

21. De Oliveira GS, Jr Castro-Alves LJ, Khan JH, McCarthy RJ. Perioperative systemic magnesium to minimize postoperative pain: a meta-analysis of randomized controlled trials. Anesthesiology 2013; 119: 178-90.

22. Morrison AP, Hunter JM, Halpern SH, Banerjee A. Effect of intrathecal magnesium in the presence or absence of local anaesthetic with and without lipophilic opioids: a systematic review and meta-analysis. Br J Anaesth 2013; 110: 702-12.

23. Shin HJ, Kim EY, Na HS, Kim TK, Kim MH, Do SH. Magnesium sulphate attenuates acute postoperative pain and increased pain intensity after surgical injury in staged bilateral total knee arthroplasty: a randomized, double-blinded, placebo-controlled trial. Br J Anaesth 2016; 117: 497-503.

24. Fawcett WJ, Haxby EJ, Male DA. Magnesium: physiology and pharmacology. Br J Anaesth 1999; 83: 302-20.

25. James $M F$, Beer RE, Esser JD. Intravenous magnesium sulfate inhibits catecholamine release associated with tracheal intubation. Anesth Analg 1989; 68: 772-6.

26. Cairns BE, Svensson P, Wang K, et al. Activation of peripheral NMDA receptors contributes to human pain and rat afferent discharges evoked by injection of glutamate into the masseter muscle. J Neurophysiol 2003; 90: 2098-105.

27. McRoberts JA, Coutinho SV, Marvizon JC, et al. Role of peripheral N-methyl-D-aspartate (NMDA) receptors in visceral nociception in rats. Gastroenterology 2001; 120: 1737-48.

28. Alfredson $H$, Lorentzon $R$. Chronic tendon pain: no signs of chemical inflammation but high concentrations of the neurotransmitter glutamate. Implications for treatment? Curr Drug Targets 2002; 3: 43-54.

29. Lawand NB, Willis WD, Westlund KN. Excitatory amino acid receptor involvement in peripheral nociceptive transmission in rats. Eur J Pharmacol 1997; 324: 169-77.

30. Carlton SM, Zhou S, Coggeshall RE. Evidence for the interaction of glutamate and NK1 receptors in the periphery. Brain Res 1998; 790: $160-9$

31. Moher D, Liberati A, Tetzlaff J, Altman DG, PRISMA Group. Preferred reporting items for systematic reviews and metaanalyses: the PRISMA statement. BMJ 2009; 339: b2535.

32. Chen KT, Tzeng JI, $\mathrm{Lu} C L$, et al. Risk factors associated with postoperative sore throat after tracheal intubation: an evaluation in the postanesthetic recovery room. Acta Anaesthesiol Taiwan 2004; 42: 3-8.

33. Piriyapatsom A, Dej-Arkom S, Chinachoti T, Rakkarnngan J, Srishewachart $P$. Postoperative sore throat: incidence, risk factors, and outcome. J Med Assoc Thai 2013; 96: 936-42.

34. Minamiguchi M, Tanaka $Y$, Kitagawa K, Inoue S, Kawaguchi $M$, Kirita T. Evaluation of factors associated with postoperative sore throat (Japanese). Masui 2014; 63: 401-5.

35. Kim E, Yang SM, Kwak SG, Park S, Bahk JH, Seo JH. Tracheal tubes lubricated with water to reduce sore throat after intubation: a randomized non-inferiority trial. PLoS One 2018; 13: e0204846.

36. Higgins JP, Green S. Cochrane Handbook for Systematic Reviews of Interventions. 1st ed. Chichester, England: John Wiley \& Sons, Ltd; 2008 .
37. Higgins JP, Altman DG, Gotzsche PC, et al. The Cochrane Collaboration's tool for assessing risk of bias in randomised trials. BMJ 2011; 343: d5928.

38. Sweeting MJ, Sutton AJ, Lambert PC. What to add to nothing? Use and avoidance of continuity corrections in meta-analysis of sparse data. Stat Med 2004; 23: 1351-75.

39. DerSimonian R, Laird N. Meta-analysis in clinical trials. Control Clin Trials 1986; 7: 177-88.

40. Higgins JP, Thompson SG, Deeks JJ, Altman DG. Measuring inconsistency in meta-analyses. BMJ 2003; 327: 557-60.

41. Wetterslev J, Jakobsen JC, Gluud C. Trial Sequential Analysis in systematic reviews with meta-analysis. BMC Med Res Methodol 2017; 17: 39.

42. Jackson JL, Kuriyama A. From the editors' desk: bias in systematic reviews-let the reader beware. J Gen Intern Med 2018; 33: 133-5.

43. Borazan H, Kececioglu A, Okesli S, Otelcioglu S. Oral magnesium lozenge reduces postoperative sore throat: a randomized, prospective, placebo-controlled study. Anesthesiology 2012; 117: 512-8.

44. Ashwini H, Seema Kumari K, Lavanya R. Comparative study of dexamethasone nebulisation with magnesium sulphate nebulisation in preventing post operative sore throat following endotracheal intubation. Indian J Clin Anaesth 2018; 5: 341-7.

45. Gupta SK, Tharwani S, Singh DK, Yadav G. Nebulized magnesium for prevention of postoperative sore throat. $\mathrm{Br} \mathrm{J}$ Anaesth 2012; 108: 168-9.

46. Jain $S$, Kumar $S$. A comparative study of preoperative ketamine and $\mathrm{MgSO} 4$ nebulisation for incidence of post operative sore throat after endotracheal intubation. IJCMR 2017; 4: 1356-9.

47. Rajan S, Malayil GJ, Varghese R, Kumar L. Comparison of usefulness of ketamine and magnesium sulfate nebulizations for attenuating postoperative sore throat, hoarseness of voice, and cough. Anesth Essays Res 2017; 11: 287-93.

48. Teymourian H, Mohajerani SA, Farahbod A. Magnesium and ketamine gargle and postoperative sore throat. Anesth Pain Med 2015; 5: e22367.

49. Yadav M, Chalumuru N, Gopinath R. Effect of magnesium sulfate nebulization on the incidence of postoperative sore throat. J Anaesthesiol Clin Pharmacol 2016; 32: 168-71.

50. Wu X, Ji L, Wang S, Shen $R$, Guo X, Gao $Q$. Effects of prophylactic magnesium sulphate on the sore throat after tracheal extubation with a double-lumen endobronchial tube (Chinese). Modern Medical Journal 2013; 41: 474-7.

51. Shen S, Xie $Y$, Chen $Y$. Comparison of the effect of ketamine and magnesium sulfate gargle in preventing sore throat after removal of a double-lumen endobronchial tube (Chinese). Chinese Journal of General Practice 2018; 16: 188-91.

52. Lin S, Jin X, Shen S. Comparison of the effect of magnesium sulfate gargle and compound lidocaine cream smeared in preventing postoperative sore throat. Chin J Mod Appl Pharm 2016; 33: 1587-91.

53. Sharma $M$, Loyal $M K$, Purohit $S$, Maniyar F, Gupta D. Comparison of magnesium sulfate and normal saline (placebo) nebulization for prevention of postoperative sore throat in patients undergoing lumbar spine surgeries under general anaesthesia with endotracheal intubation in prone position. Int $\mathrm{J}$ Sci Res 2017; 6: 656-8.

54. Kuriyama A, Umakoshi N, Sun R. Prophylactic corticosteroids for prevention of postextubation stridor and reintubation in adults: a systematic review and meta-analysis. Chest 2017; 151: 1002-10.

55. Hu B, Bao R, Wang X, et al. The size of endotracheal tube and sore throat after surgery: a systematic review and meta-analysis. PLoS One 2013; 8: e74467.

56. Mayhood J, Cress K. Effectiveness of ketamine gargle in reducing postoperative sore throat in patients undergoing 
airway instrumentation: a systematic review. JBI Database System Rev Implement Rep 2015; 13: 244-78.

57. Tanaka $Y$, Nakayama T, Nishimori M, Tsujimura $Y$, Kawaguchi $M$, Sato $Y$. Lidocaine for preventing postoperative sore throat. Cochrane Database Syst Rev 2015; 7: CD004081.

58. Begg C, Cho M, Eastwood $S$, et al. Improving the quality of reporting of randomized controlled trials. The CONSORT statement. JAMA 1996; 276: 637-9.
59. El-Boghdadly K, Bailey CR, Wiles MD. Postoperative sore throat: a systematic review. Anaesthesia 2016; 71: 706-17.

Publisher's Note Springer Nature remains neutral with regard to jurisdictional claims in published maps and institutional affiliations. 